\title{
Origin of high elastic strain in amorphous silica nanowires
}

\author{
Yonghai Yue ${ }^{1+*}$, Kun Zheng ${ }^{2,3 \dagger}$, Lei Zhang ${ }^{2,4}$ and Lin Guo ${ }^{1 *}$
}

\begin{abstract}
An understanding of the origin of elastic strain is extremely important for both crystalline materials and amorphous materials. Owing to the lack of a long range order in their structure, it is arduous to dynamically study the elastic mechanism of amorphous materials experimentally at atomic scale compared with their crystalline counterparts. Here, the elastic deformation mechanism of amorphous silica nanowires (NWs) has been studied for the first time via in situ elastic tensile tests in a transmission electron microscope. Radial distribution functions (RDFs) calculated from the corresponding selected area electron diffraction patterns (SAEDPs) at different strains were used to reconstruct a structural model based on the reverse Monte-Carlo (RMC) method. The result interestingly indicates that the elastic strain of silica glass NWs can be mainly attributed to the elastic elongation of the bond length accompanied by a change in the bond angle distribution. This work is useful for understanding the high strength of amorphous materials.
\end{abstract}

The two most crucial properties of a structural material are its elastic strain limit and plasticity. Plasticity is the property indicating the flexibility of a material, while the elastic strain limit represents the maximum stress that the material can withstand before yielding. The development of materials with high elastic strain limit and excellent plasticity is an urgent target for researchers [1-4]. Amorphous materials such as oxide glasses and metallic glasses are widely used in military applications. Unlike for their crystalline counterparts, there is still inadequate comprehension of the extraordinary behaviors of such glasses [5-10]. In nano-sized objects, smaller is stronger, owing to the combination of their decreased defect density and increased strength and elastic strain. Experimental elastic strain limits of more than $7.8 \%$ for $\mathrm{ZnO}$ nanowires (NWs) [11,12], 7.1\% for Si NWs [13], and 7.2\% for copper NWs [14] have been reported, which can be attributed to the recoverable lattice spacing elongation of these materials. Surface imperfections and larger flaws in glasses greatly affect their ultimate strength and elastic strain [15]. Reducing the size of such glasses minimizes flaw size and maximizes their strength (i.e., elastic strain). Elimination of extrinsic flaws and decreased internal structural defects can lead to an outstanding large elastic strain limit of more than 3-6\% for submicro-sized glasses [16-19], in which shear bands are suppressed owing to the reduction in size [16]. The ultimate strength of silica NWs is higher than $10 \mathrm{GPa}$ and increases with decreasing NW diameter. The reduced possibility of having large cracks in small samples is the main reason for their high strength [20]. Molecular dynamic simulations also indicate such a size-dependent elasticity for amorphous silica NWs originating from coupling between core softening and surface stiffening of each NW the structure of which is different from its bulk counterpart [21]. In our systematic studies, an approximately $13 \%$ elastic strain limit for silica NWs with diameters around $50 \mathrm{~nm}$ was confirmed. The uniaxial tensile stress corresponding to such an elastic strain limit would be $9.5 \mathrm{GPa}$ (assuming a Young's modulus of $73 \mathrm{GPa}$ for silica NWs [22]). Recent results have revealed that the movement of atomic clusters (or atoms) and open volumes directly results in large elastic strain [19]. However, the elastic mechanism of amorphous silica NWs has not been investigated experimentally at atomic scale and is still unclear.

To address the above issue and understand the performance of such materials well, it is vital to find a way to reveal the elastic mechanism of amorphous materials at atomic scale as is already possible for their crystalline counterparts. However, unlike crystalline materials, the elastic strain limit of amorphous materials cannot be evaluated according to the changes in "lattice" spacing. Poulsen and coworkers [23] successfully extracted strain data from amorphous materials with the help of a pair-distribution-function (PDF) approach, which has emerged as a powerful tool for measuring the local bond lengths and the neighbor-site occupations in a wide range of materials [24]. In this work, using a home-made in situ tensile device

${ }^{1}$ School of Chemistry and Environment, Beihang University, Beijing 100191, China

${ }^{2}$ Institute of Microstructure and Property of Advanced Materials, Beijing University of Technology, Beijing 100124, China

${ }^{3}$ Beijing Key Lab of Microstructure and Property of Advanced Material, Beijing University of Technology, Beijing 100124, China

${ }^{4}$ Electron Microscopy Lab, Center of Medical and Health Analysis \& Department of Biophysics, Peking University, Beijing 100191, China

${ }^{\dagger}$ These authors contributed equally to this work.

*Corresponding authors (emails: yueyonghai@buaa.edu.cn (Yue Y); guolin@buaa.edu.cn (Guo L)) 
[14,25-32], we conducted tensile tests on silica glass in a transmission electron microscope (TEM) and calculated a series of radial distribution functions (RDFs) from the selected area electron diffraction patterns (SAEDPs) obtained at different strains. Structural models of the NW at different strains were then reconstructed based on the reverse Monte-Carlo (RMC) method. Information extracted from these models reveals that the high elastic strain is caused by elastic elongation of the bond length accompanied by the change in bond angle. The elucidation of the elastic mechanism of silica glass NWs gives us a clearer physical image of the elasto-plastic deformation mechanism of amorphous materials.

A popular glassy material, silica glass has had more attention focused on the study of its mechanical properties in recent years. In our previous investigations, silica NWs were found to exhibit a super plasticity as high as approximately $200 \%$ [33], and an elasticity of approximately $10 \%$ [25]. A strong size effect on the ultimate strength of silica NWs was also reported [20]. Our systematic experiments in which the elastic strain limit of silica glass NWs was approached confirmed an approximately $13 \%$ elastic strain limit for silica NWs with diameters around $50 \mathrm{~nm}$ (see Fig. S2). Thus, in this work we deliberately limited the strain far below this value to ensure that our attention would be concentrated on the elastic region of the sample. A home-made bimetallic tensile device [14,25-32] was used to carry out the elastic tensile tests. The silica NWs used in the tests were fabricated with the popular thermal evaporation fabrication method [34]. The NWs were randomly spread across the two plates of the home-made bimetallic plate tensile device. A tensile pulling force was generated by heating the bimetallic sheets in a conventional TEM heating holder, to a temperature lower than $60^{\circ} \mathrm{C}$ (see Fig. S1). The real-time evolution and the deformation of the sample were captured in situ in a JEOL-2010 TEM operated at $200 \mathrm{kV}$. Figs la and $1 \mathrm{~b}$ demonstrate the TEM images taken during the tensile test. Fig. 1c is an magnified TEM image of the red dashed frame in Fig. 1a; the diameter of the NW shown is about $33 \mathrm{~nm}$. Figs $1 \mathrm{~d}$ and e show the SAEDPs taken from the red dashed frame region of Fig. 1a at different strains. The two black arrows denote the gauge length, which is used to calculate the total strain. The final strain in Fig. $1 \mathrm{~b}$ is calculated to be $7.4 \%$, much lower than the elastic strain limit of approximately $13 \%$. Combining this information with the RDFs calculated from the SAEDPs, more details can be obtained to reveal the deformation mechanism. However, it is extremely difficult to conduct the above tests, and there are several points we should clarify. First, the as-prepared silica NWs were tangled together, it was difficult to find a single NW with its ends bridged across the two bimetallic chips, and the NWs were pulled out regularly. The diameter of the NWs was another issue; a diameter of around $30 \mathrm{~nm}$ was required to ensure that the SAEDPs collected were of sufficiently high quality for RDF calculation. The intensity of the transmission signal would be very high and even cover the diffraction signals at very small NW diameters, whereas inelastic scattering information would conceal the information we need, if the diameter is very large. These are the reasons why no dynamic experimental study of the structural evolution of amorphous materials has been carried out until now.

Unlike its crystalline counterpart, we cannot attribute the high elastic strain to recoverable lattice spacing elongation because the amorphous material lacks long range order. We need a new experimental method to reveal the atomic mechanism of high elastic strain in amorphous materials. RDFs [35-37], calculated from the SAEDPs of the amorphous material, will help us to understand its elastic mechanism. In this work, the SAEDPs were calibrated using gold Debye-Scherrer rings and recorded with a Gatan 782 CCD camera, which has a wider dynamic range and a higher linearity with the electron beam intensity than conventional TEM films. The RDFs were calculated from the

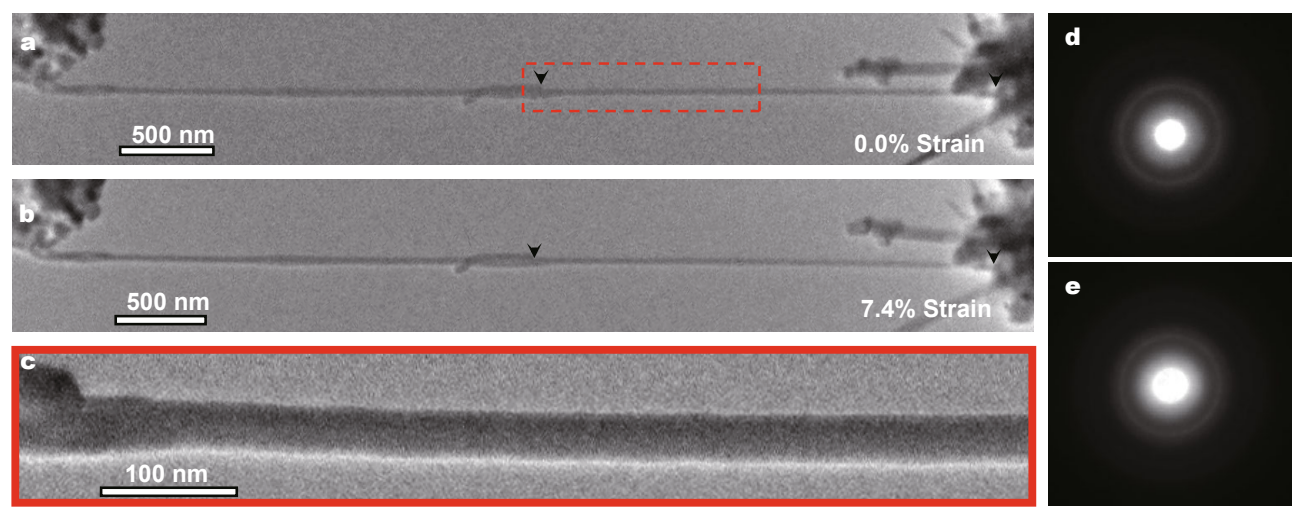

Figure 1 In situ deformation of a single silica NW at different total strains: (a) $0 \%$ strain, (b) 7.4\% strain; (c) magnified TEM image taken from the red dashed frame in (a); (d) and (e) corresponding selected area electron diffraction patterns at the different strains. 
SAEDP intensity profiles. To minimize electron beam irradiation, the NWs were only exposed to the electron beam when the images and the SAEDPs were captured, and the electron beam was shut off for the remainder of the tensile process [25]. Based on the RDFs calculated from the in situ SAEDPs of the materials, and with the help of RMC simulations, structural models could be reconstructed. Finally, detailed atomic scaled information could be extracted from the structural models, which actually came from the samples themselves. This method expands the functions of TEM to reveal structural evolution information for amorphous materials at atomic scale. The red and gray curves (experimental RDF curves) in Fig. 2a show the RDFs (reduced radial distribution function here) of the NW during the tensile test, taken from the SAEDPs at $0 \%$ and $7.4 \%$ strain. These two experimental RDF curves contain information on the distributions of coordination distances between different atoms in the NW. The first peak is the first neighbor distance, also called the nearest neighbor distance; the second peak is the second neighbor distance and the next nearest neighbor distance; and so on. It is notable that the nearest neighbor distance (the first peak) roughly corresponds to the bond length of the neighboring atoms. Thus, the shifts of the peak positions in the RDFs induced by tensile strain indicate a change in structure. Variation of the first peak will reflect changes in bond length for bonds including $\mathrm{Si}-\mathrm{O}, \mathrm{O}-\mathrm{O}$, and $\mathrm{Si}-\mathrm{Si}$. With the assistance of RMC simulations $[38,39]$, we could further reveal the changes in structure occurred during the deformation process. Based on the RDF data obtained from the SAEDPs through diffraction theory, we were able to reconstruct structural models of the NW. Figs $2 b$ and $c$ show the reconstructed models for the NW. The simulation box contains
3000 atoms (1000 silicon and 2000 oxygen), with dimensions of $17.32 \mathrm{~nm}(x) \times 17.32 \mathrm{~nm}(y) \times 17.32 \mathrm{~nm}(z)$. The number density is $72.14 \mathrm{~nm}^{-3}$. The cutoff values are 2.90 , 1.4 , and 2.35 for $\mathrm{Si}-\mathrm{Si}, \mathrm{Si}-\mathrm{O}$, and $\mathrm{O}-\mathrm{O}$, respectively. Coordination constraints and average coordination constraints are not applied to the simulations. According to the atomic ratio of $\mathrm{SiO}_{2}$, the coefficients for partial pair distribution functions are $0.1667,0.2222$ and 0.3333 for $\mathrm{Si}-\mathrm{Si}, \mathrm{Si}-\mathrm{O}$, and $\mathrm{O}-\mathrm{O}$, respectively. When the simulated curves and experimented curves had been matched as closely as possible, the simulations finished in convergence states and equilibrium states. The resulting models could be used to recalculate the RDFs, i.e., simulated RDFs curves. The models were adjusted until the simulated RDFs curves fitted well with the original RDFs calculated from the SAEDPs at different strains. The black and blue curves in Fig. 2a show the obtained RMC simulation curves, which are consistent with the experimental results. Thus, the obtained models represent the real structure of the NW.

Fig. 3 shows the vibrations of $\mathrm{Si}-\mathrm{O}, \mathrm{O}-\mathrm{O}$, and $\mathrm{Si}-\mathrm{Si}$ bond lengths during the tensile test at different strains. The $\mathrm{Si}-\mathrm{O}$ bond length is the critical parameter that represents the change in the structure of $\mathrm{SiO}_{4}$ tetrahedrons. As shown in Fig. 3a, we can see the original average $\mathrm{Si}-\mathrm{O}$ bond length is about $1.61 \AA$, in good accordance with previous results [40-42]. The bond length increases with deformation to about $1.64 \AA$ at a total strain of $7.4 \%$. The spectrum becomes sharp and the intensity increases at the same time, which indicates that the order of the atomic arrangement inside the NW increases. Changes in the $\mathrm{Si}-\mathrm{Si}$ and $\mathrm{O}-\mathrm{O}$ bonds are also extracted from the structure (Figs $3 \mathrm{~b}$ and c). The first O-O peak shifts from $2.60 \AA$ in the pre-test specimen to $2.68 \AA$ in the specimen at $7.4 \%$ strain. The first
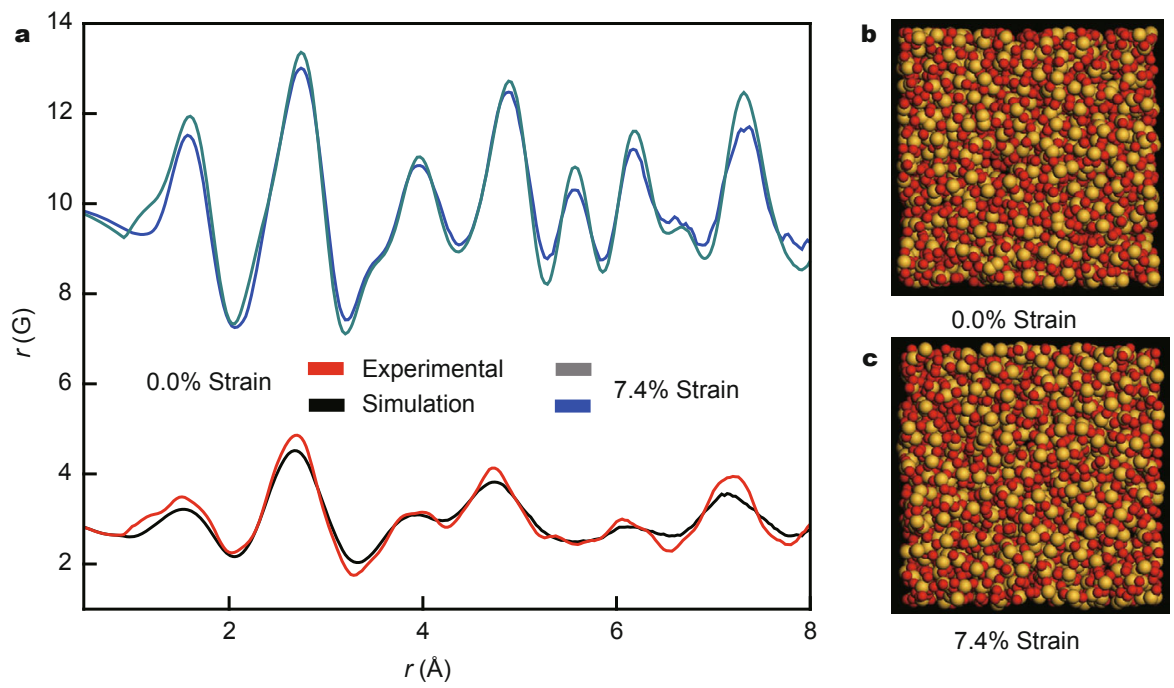

Figure 2 (a) Experimental (red, 0.0\% strain and gray, 7.4\% strain) and reverse Monte-Carlo simulation (black, $0.0 \%$ strain and blue, 7.4\% strain) radial distribution function curves of the NW during the tensile test; (b) and (c) reconstructed models at different strains of the silica NW. 

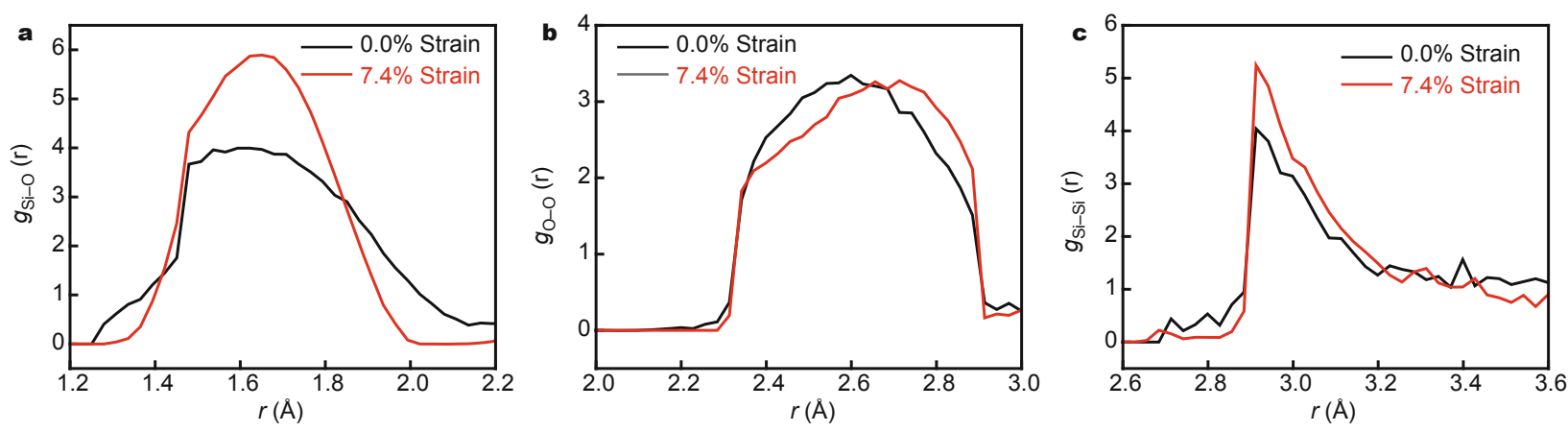

Figure $3 \mathrm{Si}-\mathrm{O}, \mathrm{O}-\mathrm{O}$, and $\mathrm{Si}-\mathrm{Si}$ pair-distribution functions.

Si-Si peak also shifts to higher bond length, from $2.94 \AA$ to $2.97 \AA$. This result is attributed to the accumulation of small increases in bond length on the atomic scale, which gives rise to the tension on the bulk scale. However, such tiny strain (approximately $1.9 \%$ strain, taking the change in $\mathrm{Si}-\mathrm{O}$ bond length from $1.61 \AA$ to $1.64 \AA$ as an example) is far lower than the total elastic strain limit mentioned above (the total elastic strain of silica glass can be above 10\% [25], and we have actually observed 13\%, as shown in Fig. S2), so there must be other factors that contribute to the total elastic strain in cooperation with the bond length.

The microscopic structural evolution under tension was further analyzed in terms of angular correlations between atoms. The $\mathrm{Si}-\mathrm{O}-\mathrm{Si}$ and $\mathrm{O}-\mathrm{Si}-\mathrm{O}$ angle distributions during tensile deformation were extracted as shown in Figs $4 \mathrm{a}$ and b. As previously reported [40-42], there is a broad range of inter-tetrahedral $\mathrm{Si}-\mathrm{O}-\mathrm{Si}$ angles, from $120^{\circ}$ to $180^{\circ}$, with a most probable value of approximately $144^{\circ}$. Here, the $\mathrm{Si}-\mathrm{O}-\mathrm{Si}$ angle distribution of the pre-test specimen shows a peak centered at $135^{\circ}$ with a full width at half maximum (FWHM) of $54^{\circ}$. With increasing strain, this peak finally moves to $151^{\circ}$ and exhibites a narrowed FWHM of $43^{\circ}$ at $7.4 \%$ strain, indicating a slight distortion of single tetrahedron and also the network at high strain. This shift in the distribution by $16^{\circ}$ combines with the $0.03 \AA$ elongation in the $\mathrm{Si}-\mathrm{O}$ bond length to contribute to the total strain. In contrast to the huge change in $\mathrm{Si}-\mathrm{O}-\mathrm{Si}$ bond angle distribution, there is only a subtle change in the $\mathrm{O}-\mathrm{Si}-\mathrm{O}$ angle distribution, as shown in Fig. $4 \mathrm{~b}$. The peak shifts from $105^{\circ}$ (no strain) to $108^{\circ}$ (7.4\% strain), and the FWHM shrinks from $38^{\circ}$ to $29^{\circ}$. Fig. $4 \mathrm{c}$ shows a brief sketch of two adjacent $\mathrm{SiO}_{4}$ tetrahedrons, which shows the change before and after the tensile test. We take the maximum average angle before and after the tensile test as $\theta$ and $\theta^{\prime}$ as marked in Fig. $4 \mathrm{c}$, and we also suppose that the direction of elongation of these two $\mathrm{SiO}_{4}$ tetrahedrons is parallel to the loading direction. It is widely accepted $[43,44]$ that silica can be taken as a network of $\mathrm{SiO}_{4}$ tetrahedrons, in which each $\mathrm{Si}$ atom is connected to four $\mathrm{O}$ atoms. These $\mathrm{SiO}_{4}$ tetrahedrons form nanometer sized -Si-O-Si-O-Si- rings with corner-sharing $\mathrm{O}$ atoms [45]. The network has an extremely flexible local atomic structure and significant variability in $\mathrm{Si}-\mathrm{O}-\mathrm{Si}$ bond angles [46]. Here, if we take the maximum changes in both the bond length and the bond angle, $1.9 \%$ and $16^{\circ}$, respectively, based on the cosine theorem, we can roughly calculate the distance of the two tetrahedral centers to finally obtain a strain value of approximately $6.8 \%$ (as shown in Fig. 4c), which is approaching the total elastic strain. In addition, the change in ring structure caused by the changes in bond angle distribution and bond length will
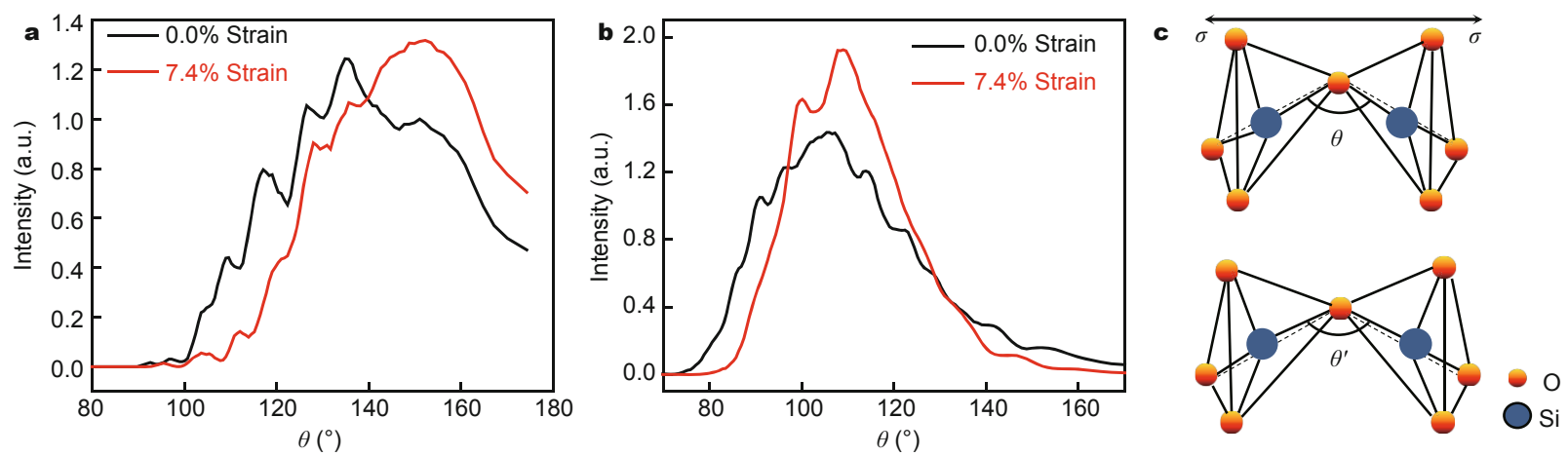

Figure 4 (a) Si-O-Si bond angle distribution at different strains; (b) O-Si-O bond angle distribution at different strains; (c) sketch map showing the change for two $\mathrm{SiO}_{4}$ tetrahedrons. 
undoubtedly contribute to the total elastic strain. Besides these two main factors, ring structure is another factor we should take into account.

With increasing strain, the contribution from the change in bond length will be slightly less than that from the bond angle distribution. Because of the random location of $\mathrm{SiO}_{4}$ tetrahedrons, there is no lattice inside the silica NW and thus a lack of long range order, so the $\mathrm{Si}-\mathrm{O}$ bonds cannot be elongated along the same direction as which can occur in crystal materials. As a result, the bond length will exhibit little elongation compared with that in single crystal materials [14]. Change in the $\mathrm{Si}-\mathrm{O}-\mathrm{Si}$ bond angle distribution will compensate for this small contribution from variation in bond length. Furthermore, the ring structure can be affected by the change in bond angle distribution. Upon elastic deformation, the puckered ring will tend to become planar-like along the tension direction, resulting in further increase in the elastic strain.

In conclusion, via in situ tensile tests in a TEM, the origin of the high elastic strain of silica glass NWs has been studied for the first time. RDFs calculated based on the SAEDPs taken during the in situ tensile test were used to reconstruct structural models of the NWs using an RMC method. More detailed atomic scale information was extracted from the reconstructed models of the tensile NWs. The results indicate that the high elastic strain of silica NWs is not dependent on recoverable lattice spacing elongation, but originates mainly from the coupling of elastic elongation of bonds and changes in bond angle distribution. Changes in the ring structure also assist the elastic deformation of the material. The elastic deformation mechanism revealed in this work is different from those of crystalline materials. The detailed information that we extracted from the reconstructed structural models sheds light on the elastic deformation mechanism of amorphous materials at atomic scale. Our results may have implications for the processing and functions of amorphous materials as they are scaled down to the nanometer regime in applications.

Received 2 March 2015; accepted 30 March 2015; published online 20 April 2015

1 Lu L, Shen Y, Chen X, et al. Ultrahigh strength and high electrical conductivity in copper. Science, 2004, 304: 422-426

2 Smith DA, Holmberg VC and Korgel BA. Flexible germanium nanowires: ideal strength, room temperature plasticity, and bendable semiconductor fabric. ACS Nano, 2010, 4: 2356-2362

3 Deng $\mathrm{C}$ and Sansoz F. Near-ideal strength in gold nanowires achieved through microstructural design. ACS Nano, 2009, 3: 3001-3008

4 Meza LR, Das S, Greer JR. Strong, lightweight, and recoverable three-dimensional ceramic nanolattices. Science, 2014, 345: 1322-1326

5 Greer AL, Ma E. Bulk metallic glasses: at the cutting edge of metals research. MRS Bull, 2007, 32: 611-615
6 Busch R, Schroers J, Wang WH. Thermodynamics and kinetics of bulk metallic glass. MRS Bull, 2007, 32: 620-623

7 Inoue A, Nishiyama N. New bulk metallic glasses for applications as magnetic-sensing, chemical, and structural materials. MRS Bull, 2007, 32: 651-658

8 Sheng HW, Luo WK, Alamgir FM, et al. Atomic packing and shortto-medium-range order in metallic glasses. Nature, 2006, 439: 419-425

9 Schuh CA, Hufnagel TC, Ramamurty U. Overview of No.144-mechanical behavior of amorphous alloys. Acta Mater, 2007, 55: 4067-4109

10 Wang WH. The elastic properties, elastic models and elastic perspectives of metallic glasses. Prog Mater Sci, 2012, 57: 487-656

11 Wei B, Zheng K, Ji Y, et al. Size-dependent bandgap modulation of ZnO nanowires by tensile strain. Nano Lett, 2012, 12: 4595-4599

12 Shao RW, Zheng K, Wei B, et al. Bandgap engineering and manipulating electronic and optical properties of $\mathrm{ZnO}$ nanowires by uniaxial strain. Nanoscale, 2014, 6: 4936-4941

13 Zhu Y, Xu F, Qin QQ, et al. Mechanical properties of vapor-liquid-solid synthesized silicon nanowires. Nano Lett, 2009, 9: 3934-3939

14 Yue YH, Liu P, Zhang Z, et al. Approaching the theoretical elastic strain limit in copper nanowires. Nano Lett, 2011, 11: 3151-3155

15 Griffith AA. The Phenomena of Rupture and Flow in Solids. London: Philos Trans R Soc, 1921, 221A: 163-198

16 Jang D, Greer JR. Transition from a strong-yet-brittle to a strongerand-ductile state by size reduction of metallic glasses. Nat Mater, 2010, 9: 215-219

17 Tian L, Chen YQ, Shan ZW, et al. Approaching the ideal elastic limit of metallic glasses. Nat Commun, 2012, 3: 609

18 Deng QS, Chen YQ, Yue YH, et al. Uniform tensile elongation in framed submicron metallic glass specimen in the limit of suppressed shear banding. Acta Mater, 2011, 59: 6511-6518

19 Jiang QK, Liu P, Ma Y, et al. Super elastic strain limit in metallic glass films. Sci Rep, 2012, 2: 852

20 Brambilla G, Payne DN. The ultimate strength of glass silica nanowires. Nano Lett, 2009, 9: 831-835

21 Yuan FL, Huang LP. Size-dependent elasticity of amorphous silica nanowire: a molecular dynamics study. Appl Phys Lett, 2013, 103: 201905

$22 \mathrm{Ni} \mathrm{H}, \mathrm{Li} \mathrm{XD}$, Gao HS. Elastic modulus of amorphous $\mathrm{SiO}_{2}$ nanowires. Appl Phys Lett, 2006, 88: 043108

23 Poulsen HF, Wert JA, Neuefeind J, et al. Measuring strain distributions in amorphous materials. Nat Mater, 2005, 4: 33-36

24 Egami T, Billinge SJL. Underneath the Bragg Peaks: Structural Analysis of Complex Materials. Oxford: Elsevier, 2003, 7

25 Yue YH, Zheng K. Strong strain rate effect on the plasticity of amorphous silica nanowires. Appl Phys Lett, 2014, 104: 231906

26 Han XD, Zhang YF, Zhang Z. A grid of transmission electronic microscope driven by thermal dual metal sheets. Chinese Patent, No. 200610144031. x, 2009-06-03

27 Han XD, Yue YH, Zheng K, et al. Stress test grid of nano material used for transmission electron microscopy. Chinese Patent, No. 200810056836. 8, 2010-02-03

28 Han XD, Zhang YF, Zhang Z. Nano-wire in-situ stretching device in scanning electron microscope and method therefor. Chinese Patent, No. 200610169839. 3, 2010-02-17

29 Wang LH, Han XD, Liu P, et al. In situ observation of dislocation behavior in nanometer grains. Phys Rev Lett, 2010, 105: 135501

30 Yue YH, Liu P, Deng QS, et al. Quantitative evidence of crossover toward partial dislocation mediated plasticity in copper single crystalline nanowires. Nano Lett, 2012, 12: 4045-4049

31 Yue YH, Wang LH, Zhang Z, et al. Cross-over of the plasticity mechanism in nanocrystalline Cu. Chinese Phys Lett, 2012, 29: 066201

32 Yue YH, Chen NK, Li XB, et al. Crystalline liquid and rubber-like 
behavior in Cu nanowires. Nano Lett, 2013, 13: 3812-3816

33 Zheng K, Wang CC, Cheng YQ, et al. Electron-beam-assisted superplastic shaping of nanoscale amorphous silica. Nat Commun, 2010, 1: 24

34 Qin Y, Zhang XN, Zheng K, et al. Synthesis and photoluminescence of amorphous $\mathrm{SiO}_{2}$ nanowires. J Chin Electron Microsc Soc, 2008, 27: 102-107

35 Lannin JS. Structural order and dynamics of amorphous Si and Ge. J Non-Cryst Solids, 1987, 97/98: 39-46

36 Laaziri K, Kycia S, Roorda S, et al. High resolution radial distribution function of pure amorphous silicon. Phys Rev lett, 1999, 82: 3460-3463

37 Zhang L, Han XD, Zhang Z. Crystallization process of in situ annealed $\mathrm{Ge}_{2} \mathrm{Sb}_{2} \mathrm{Te}_{5}$ films. J Alloys Compounds, 2012, 537: 71-75

38 McGreevy RL, Pusztai L. Reverse Monte Carlo simulation: a new technique for the determination of disordered structures. Molec Simul, 1988, 1: 359-367

39 Yan N, Zhang L, Zhang Z, et al. The amount of Ge tunes the atomic structure of amorphous $\mathrm{Ge}_{x} \mathrm{Te}_{1-x}$ alloy. Chemical Physics Letters, 2013, 556: 108-112

40 Mozzi RL, Warren BE. The structure of vitreous silica. J Appl Crystallogr, 1969, 2: 164-172

41 Sinclair RN, Desa JAE, Etherington G, et al. Neutron diffraction studies of amorphous solids. J Non-Cryst Solids, 1980, 42: 107-115

42 Coombs PC, De Natale JF, Hood PJ, et al. The nature of the Si-O-Si bond angle distribution in vitreous silica. Philos Mag B, 1985, 51: L39-L42

43 Wright AC. Neutron scattering from vitreous silica. V. The structure of vitreous silica: what have we learned from 60 years of dif- fraction studies? J Non-Cryst Solids, 1994, 179: 84-115

44 Wright AC, Clare AG, Grimley DI, et al. Neutron scattering studies of network glasses. J Non-Cryst Solids, 1989, 112: 33-47

45 Chen $\mathrm{YC}, \mathrm{Lu} \mathrm{Z}$, Nomura $\mathrm{K}$, et al. Interaction of voids and nanoductility in silica glass. Phys Rev lett, 2007, 99: 155506

46 Kingery WD, Bowen HK, Ulhmann DR. Introduction to Ceramics (2nd ed.). New York: Wiely-Interscience, 1976, 1032

Acknowledgements This work was supported by the National Natural Science Foundation of China (51301011 and 11374029), Research Fund for the Doctoral Program of Higher Education of China (20131102120053), Fundamental Research Funds for the Central Universities (YWF-14-HHXY-013), Foundation for the Author of National Excellent Doctoral Dissertation of China (201214), Beijing Nova Program (Z121103002512017), Key Project of National Natural Science Foundation of China (11234011), Henry Fok of the Ministry of Education Fund (141008) and Beijing City Board of Education Project (KM201310005009).

Author contributions Yue Y and Guo L designed the experiments, Yue $\mathrm{Y}$ and Zheng $\mathrm{K}$ performed the in situ tensile experiments, Zhang L performed the simulation. Yue Y, Zheng K, and Guo L wrote the paper. All authors contributed to the general discussion.

Conflicts of interest The authors declare that they have no conflicts of interest.

Supplementary information Experimental details and supporting data are available in the online version of the paper. 


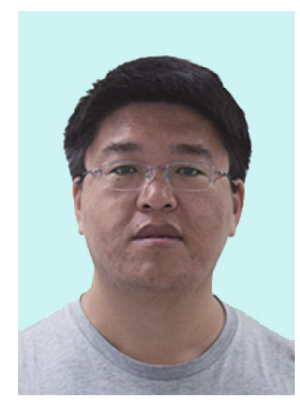

Yonghai Yue received his $\mathrm{PhD}$ degree in materials science from Beijing University of Technology under the supervision of Prof. Xiaodong Han in 2012. He received Beijing Excellent Doctoral Dissertation prize in 2013. He is currently a lecturer at the School of Chemistry and Environment of Beihang University. His research interests focus on the characterization of nanomaterials, in situ mechanical study of nanomaterials, and new in situ SEM and TEM techniques. He has published more than 20 peer-reviewed papers in this field and has more than 30 patents authorized.

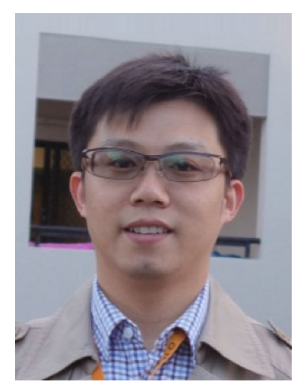

Kun Zheng received his PhD degree from Beijing University of Technology in 2009 and received the National Excellent Doctoral Dissertation of China prize in 2011. He is currently a professor at Beijing University of Technology. His research focuses on in situ TEM. He has published more than 50 peer-reviewed papers with approximately 1100 citations.

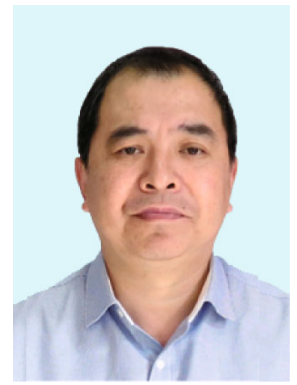

Lin Guo received his $\mathrm{PhD}$ in materials science and engineering from Beijing University Institute of Technology (BIT) in 1997. He spent 20 months as a postdoctoral research assistant at the Institute of High Energy Physics, Chinese Academy of Sciences. He worked as a visiting scholar in Prof. Shihe Yang's lab at Hong Kong University of Science and Technology (HKUST) in 1999. He then went to Dresden Technology University for 2 years on a Humboldt Fellowship. $\mathrm{He}$ is currently a professor and vice dean of the School of Chemistry and Environment at Beihang University. His research interests include the synthesis and characterization of sophisticated nanomaterials, high-strength nanomaterials with light weight, and functional nanomaterials for energy storage. He currently has more than 170 publications in this field.

中文摘要 对弹性应变起源的理解对于晶体材料或非晶材料来说都非常重要. 然而人们对非晶变形的认知远远落后于对晶体的认知. 本文采用在透射电镜中实施非晶氧化硅纳米线的原位单轴弹性拉伸, 获得一系列拉伸过程中的电子衍射谱, 通过计算它们的径向分布 函数, 结合逆蒙塔卡罗方法实现结构的重构, 从中提取出原子尺度的结构变化信息. 结果显示, 氧化硅非晶纳米线的弹性应变主要来源 于键长的弹性伸长和键角分布的变化, 并且后者的贡献更大. 本工作将对我们正确理解非晶材料的高强度提供非常重要的帮助. 